\title{
Die Europäische Republik - so nah und wohl doch so fern?
}

Seit den Referenden in Frankreich und den Niederlanden im Frühjahr 2005 ist nicht mehr auszuschließen, dass das europäische Verfassungsprojekt zu Fall kommen wird. Aber was ist mit diesem Scheitern der Verfassung eigentlich verbunden? Scheitert die Verfassungsgebung, so Armin von Bogdandy, dann »scheitert das Projekt einer europäischen Republik als neue Qualität des europäischen Zusammenschlusses « (von Bogdandy 2005: 27). Mit dem Vergleich zur Republik stellt von Bogdandy m. E. klar heraus, dass es sich bei der Verfassungsgebung um eine klassische normative politische Idee handelt: Auch wenn der Begriff weder in der Namensgebung noch im Verfassungstext in Form eines positiven Rechtsbegriffs auftaucht, soll ein republikanisches Gemeinwesen geschaffen werden, das nicht nur die Hoheitssymbole wie Hymne, Flagge und Feiertag nutzt; hinzu kommen die Personalisierung der EU mit einem Präsidenten der Union, einem Außenminister und einem gestärkten Kommissionspräsidenten sowie die Überführung in eine republikanische Ordnung mit einem Zweikammersystem unter einer Grundrechtscharta. Bei genauerer Betrachtung des Verfassungstextes fällt sogar auf, dass der »Vertrag über eine Verfassung für Europa« wenig inhaltlich Neues schafft, und wohl eher diesem normativen Ziel folgt (König et al. 2005).

Ohne Zweifel ist die Verfassungsgebung kein isolierter Akt, sondern ein Prozess, der mehrere Ereignisse einschließt. Kennzeichnend ist die Interaktion von zwei Ebenen, der nationalen Ebene und der supranationalen Integrationsebene, auf der sich die EU mit eigener Gesetzgebung und Parlamentswahlen etabliert hat. Diese ZweiEbenen-Interaktion dürfte charakteristisch für die Integrationsgeschichte wie auch für die jüngste Verfassungschronik sein, die mit dem Laekener Konvent und einem Entwurf begann, auf den die nationale Koordinierung des Brüsseler Gipfels folgte. Stets bestimmten nationale Ereignisse den weiteren Verlauf auf der supranationalen Ebene: Der für Dezember 2003 vorgesehene Gipfelbeschluss scheiterte zunächst am Widerstand Polens und Spaniens, und erst die Abwahl der Regierung Aznar machte den Weg frei für einen Kompromiss, den die irische Präsidentschaft im Juni 2004 vorlegen konnte (König 2005). Die unpopuläre polnische Regierung Miller, die sich zuvor an den Vertrag von Nizza gebunden hatte, konnte dem Druck alleine nicht mehr standhalten, und so konnten die 25 Staats- und Regierungschefs den »Vertrag über eine Verfassung für Europa« am 29. Oktober 2004 in Rom unterzeichnen (Gwiazda 2006). Es folgten die nationalen Ratifikationsprozesse mit den bekannten Volksentscheiden, durch die das Verfassungsprojekt ironischerweise zum ersten Mal eine breite Öffentlichkeit erfuhr. 
Soll man nun die Frage beantworten, ob die Integrationstheorien nach den negativen Referenden obsolet sind, dann dürfte m. E. die Antwort »Nein« lauten. Warum sollen - ganz allgemein gesprochen - Theorien obsolet sein, wenn der Ausgang eines oder mehrerer Ereignisse eine bestimmte Ausprägung hat? Ansonsten müssten doch - auf die Integrationsgeschichte bezogen - diese Theorien schon spätestens seit den Volksabstimmungen 1991 in Dänemark über den Maastrichter Vertrag und 2000 in Irland über den Vertrag von Nizza obsolet sein. Aber was können die Integrationstheorien zur Erklärung des Prozesses beitragen? Meine Bewertung der wissenschaftlichen Erklärungskraft der Integrationstheorien fällt zurückhaltend aus, da weder Funktionalismus noch Intergouvernementalismus, weder MehrebenensystemAnalyse noch neuere sui generis-Ansätze Prognosen abgeben, an denen sich ihre Erklärungskraft bemessen ließe. So kann man auch ohne größere Anstrengung in einigen Jahren zu dem Schluss kommen, dass die heutige Verfassungskrise eine notwendige Bedingung für die weitere funktionale Entwicklung, ein kleines Missgeschick in der Rhetorik der Verhandelnden oder ein Ausdruck der Macht derjenigen Regierungen gewesen sei, die sich damals doch lieber an den Regeln des Vertrags von Nizza als an den Vorschlägen des Verfassungskonvents ausrichten wollten.

Diese Beliebigkeit erschreckt nicht nur jeden an der Integrationsforschung Interessierten. Sie macht deutlich, dass diese Literatur keine überprüfbaren Erklärungen, sondern allenfalls diskussionsanregende Beschreibungen für die Integration bereitstellt, die weder für eine ereignis- oder prozessorientierte Forschung noch für die Verwendung von Erkenntnissen geeignet sein dürften, die für die Schaffung einer Republik hilfreich sein könnten. Auch neuere Varianten haben m. E. große Schwierigkeiten, den negativen Ausgang eines Ereignisses oder Prozesses vorherzusagen, wie etwa eine Verfassungskrise oder den Ausschluss Bulgariens und Rumäniens aus der letzten Beitrittsrunde. Solche Varianten haben oft die Behauptung gemein, dass andere Ansätze keine hinreichende Erklärung für ein scheinbar komplexes Bild liefern könnten, das es mit einer noch komplexeren Variante zu erklären gilt. Spätestens an dieser Stelle dürften erkenntnistheoretische Zweifel angebracht sein, da Prognosen und Erklärungen für politische Phänomene verlangt werden, die selten auftreten und deshalb das Gesetz der kleinen Zahl zu beachten ist. Es stellt sich daher die Frage, was die politikwissenschaftliche Forschung an Ansätzen bereitstellt, die eine Erklärung für die aktuellen und zurückliegenden Ereignisse geben könnten und die sich womöglich in eine Handlungsanweisung für die Zukunft ummünzen lassen.

Vielleicht ist man bei historischen Prozessen wie der Verfassungsgebung gut beraten, anfangs möglichst einfache Fragen beantworten zu wollen und sich der Komponenten bewusst zu werden, die für deren Beantwortung zu erfassen sind. Womöglich gibt es gemeinsame Komponenten, die für verschiedene Ansätze geeignet sind und die die Vielzahl an Erklärungen überprüfen helfen, die zu den jüngsten Ereignissen in- und außerhalb der Politikwissenschaft geäußert wurden. Und im günstigen Fall trifft sogar die eine oder andere Erklärung zu oder bietet zumindest eine alternative Sichtweise auf den Prozess und die Ereignisse der Verfassungsgebung, aus der sich wiederum Rückschlüsse für die Zukunft und ein besseres 
Verständnis für das mögliche Scheitern einer Republik entwickeln ließen. Hierbei könnte auch die ideelle Zuspitzung auf den Republikbegriff helfen, die jüngste Entwicklung nicht als Überraschung, sondern vorhersehbare Krise eines normativen Ziels einzustufen, und doch festzustellen, dass Europa - auch bezüglich seiner historischen Integrationsdimension - noch niemals zuvor so einig und nahe an der Realisierung einer republikanischen Idee war.

Welche Erkenntnisse liegen uns heutzutage hierzu vor? Wie sah der Prozess der Verfassungsgebung aus? Welche Themen und Akteure bestimmten Ereignisse und Verlauf? Zur Beantwortung möchte ich auf einige Ergebnisse aus dem Forschungsprojekt »Domestic Structures and European Integration« (DOSEI) zurückgreifen, das den Prozess der Verfassungsbildung und seine einzelnen Schritte sehr detailliert untersucht (König 2005; König/Hug 2006). Es sei daran erinnert, dass (trotz schlechter Vorzeichen nach dem Vertrag von Nizza und der Osterweiterung) durch die Laekener Erklärung von 2003 ein Reformprozess angestoßen wurde, der in einen Text zu einem Verfassungsvertrag mündete. Unsere ersten Auswertungen zum Konvent zeigen, dass durchaus dieselben Konfliktlinien und -lager wie bei vorangegangen, inhaltlich enttäuschenden Regierungskonferenzen vorlagen (König et al. 2006). Wie kam also der Konvent dennoch zu einem Ergebnis? Zum einen setzten sich vor allem Delegierte - unabhängig von ihrer Nationalität - durch, die in der Nähe des Status quo lagen und große Koalitionen bilden konnten (König/Slapin 2005). Im Vergleich zum Konsensprinzip von Regierungskonferenzen mussten diese Konventskoalitionen jedoch nicht das Einstimmigkeitskriterium erfüllen. Im Gegensatz zum intergouvernementalistischen Ansatz war die Größe des Landes auch nicht entscheidend, da sich eine große Nähe zu Frankreich und Großbritannien sogar negativ auf den Delegiertenerfolg auswirkte. Zum anderen zeigt die empirische Vetospieleranalyse von George Tsebelis (2006), wie Giscard D'Estaing seine Agendasetzungsmacht nutzte und geschickt den Konvent organisierte, der schließlich einen gemeinsamen Text über einen Verfassungsentwurf beschloss. Aber welche Folgen hat(te) diese manipulative Machtausübung des Agendasetzers für den weiteren Verfassungsgebungsprozess?

Legt man die supranationalistische Perspektive von George Tsebelis und Geoffrey Garrett (2001) zugrunde, dann dürfte der Laekener Entschluss eine große Bedeutung für die folgenden Ereignisse gehabt haben, da ein Vertragsentwurf bei großer Uneinigkeit unter den Mitgliedsstaaten das Ergebnis der folgenden Regierungskonferenz bestimmen kann. Giscard D'Estaing käme dann sogar eine doppelte Agendasetzungsfunktion zu (König 2002, 2005). Wie uneinig waren sich also die Mitgliedsstaaten? Ab Herbst 2003 wurde der Entwurf in den Regierungszentralen mit Blick auf die anstehende Konferenz diskutiert, um die Positionen zu den Themen der Verfassung auszuarbeiten. Im Vergleich zu Laeken, wo noch Parlamentarier und Regierungsvertreter in gleicher Anzahl auftraten, fand diese Koordination - mit Ausnahme der skandinavischen Länder - in den Exekutiven unter Führung der Außenund Premierminister statt (Hug/König 2006). Die vergleichende DOSEI-Analyse dieser Koordinationsprozesse ergibt, dass in einigen Ländern große Differenzen bestanden, vor allem in den Beitrittsstaaten und auch in Frankreich und Deutsch- 
land, wobei eine integrationsunterstützende Öffentlichkeit das Ausmaß an kollektiver Positionsheterogenität deutlich reduzierte (Hug/König 2006).

\section{Abbildung 1: Die Positionen der Mitgliedsstaaten zu einer Europäischen Verfassung}

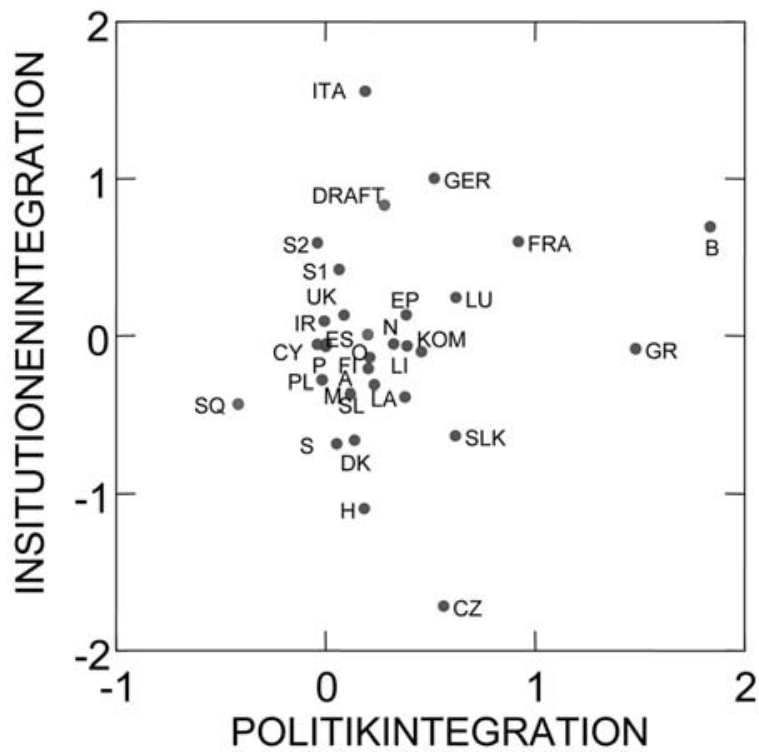

$\begin{array}{ll}\text { A: } & \text { Österreich } \\ \text { B: } & \text { Belgien } \\ \text { CY: } & \text { Zypern } \\ \text { CZ: } & \text { Tschechien } \\ \text { DK: } & \text { Dänemark } \\ \text { DRAFT: Verfassungsentwurf } \\ \text { ES: } & \text { Estland } \\ \text { EP: } & \text { Europaparlament } \\ \text { FI: } & \text { Finnland } \\ \text { FRA: } & \text { Frankreich } \\ \text { GER: } & \text { Deutschland } \\ \text { GR: } & \text { Griechenland } \\ \text { H: } & \text { Ungarn } \\ \text { IR: } & \text { Irland } \\ \text { ITA: } & \text { Italien } \\ \text { KOM: } & \text { Kommission } \\ \text { LA: } & \text { Lettland } \\ \text { LI: } & \text { Litauen } \\ \text { LUX: } & \text { Luxemburg } \\ \text { M: } & \text { Malta } \\ \text { N: } & \text { Niederlande } \\ \text { O: } & \text { Verfassungstext } \\ \text { P: } & \text { Portugal } \\ \text { PL: } & \text { Polen } \\ \text { S1: } & \text { Spanien unter Aznar } \\ \text { S2: } & \text { Spanien unter Zapatero } \\ \text { S: } & \text { Schweden } \\ \text { SL: } & \text { Slowenien } \\ \text { SLK: } & \text { Slowakei } \\ \text { SQ: } & \text { Status Quo nach Nizza } \\ \text { UK: } & \text { Großbritannien } \\ & \end{array}$

Anmerkungen: Methode: 2-Parametrisches Item Response Model mit gemischtem MetropolisHasting-/Ghibbs-Sampling Algorithmus. Konvergenz nach ca. 200 Iterationen, Probit-Link, programmiert in GAUSS 5.0. Verwendet wurden alle Positionen der 27 kollektiven Akteure zu den insgesamt 65 Themen, die im Rahmen des DOSEI-Projekts vor dem Gipfelbeschluss erfasst wurden; Institutionenintegration: Präsidentschaft, Wahl des Ratspräsidenten, Bestimmung der Kommissare, Außenminister, Initiativrecht des EP, Migration, Außengrenzen, Gemeinsame Verteidigungspolitik; Politikintegration: alle Politikfelder (außer Agrarpolitik und Strukturfonds) hinsichtlich Umfang - Abstimmungsregel und Mitbestimmung des EP, Ziele der Union, Abstimmungsregeln bei Qualified Majority Voting, Initiativrecht der Bürger, Stabilitätspakt.

Zur Veranschaulichung der Interessenlage illustriert Abbildung 1 die Positionen der Gipfelakteure, die auf zwei grundlegende Themenkomplexe reduziert und in Bezug zu dem durch den Vertrag von Nizza festgelegten Status quo (SQ), zum Laekener Entwurf (DRAFT) und zum endgültigen Verfassungstext (O) gestellt sind. Auf der horizontalen Achse sind Positionen zur Politik-, auf der vertikalen Achse zur Institutionenintegration abgebildet. Je häufiger ein Akteur für die Politikintegration eintrat, desto weiter rechts, je häufiger eine ungleiche Machtverteilung favorisiert wurde, desto weiter oben ist dieser in der Abbildung lokalisiert. Konkret zeigt sich, dass sich 
die Mitgliedsstaaten unisono für eine Integration von weiteren Politikfeldern aussprechen, wenn auch Unterschiede über das Ausmaß bestehen. Ganz anders sieht die Situation auf der Institutionendimension aus, wo sich Konflikte vor allem zwischen kleinen neuen und großen alten Staaten um den Zugang zur Kommission und die Stimmenverteilung im Ministerrat abzeichnen. Konnten die kleinen Mitgliedsstaaten noch über ein Zugeständnis bei der Besetzung der Kommission besänftigt werden, drohte die Regierungskonferenz tatsächlich wegen des Streits über die zukünftige Mehrheitsregel im Ministerrat am Widerstand Polens und Spaniens zu scheitern. Auch diese Situation lässt sich aus Abbildung 1 ablesen, die den Kompromiss (O) als eine für alle Akteure akzeptable Lösung anzeigt. Die Unterzeichnung des Vertragstextes war jedoch nur eine von vielen Etappen auf dem Weg zu einer Verfassung, die anschließend sehr hohe Hürden der Ratifikation in allen Mitgliedsstaaten nehmen muss (Hug/Schulz 2005). Da in vielen Ländern die Ratifikationshürde den hohen qualifizierten Erfordernissen für Verfassungsänderungen entspricht und eine unerwartet hohe Zahl von zehn Staaten Referenden über den Vertragstext ankündigte, sind laut Zwei-Ebenen-Ansatz Rückwirkungen auf das Ergebnis zu erwarten.

Vor den Referenden in Frankreich und den Niederlanden hatten bereits neun Staaten den Vertrag ratifiziert; die Parlamente von Lettland (2. Juni), Zypern (30. Juni) und Malta (6. Juli) wie auch die Luxemburger (10. Juli) per Volksentscheid folgten danach, während Polen, Dänemark, Portugal, Irland, die Tschechische Republik, Schweden und Großbritannien die Ratifikation aussetzten. Besonders die Volksabstimmungen in Großbritannien und Polen dürften sehr große Hürden darstellen, denn erste vergleichende Untersuchungen von Volksabstimmungen und parlamentarischen Ratifikationen suggerieren, dass Bevölkerungen gegenüber Parlamentariern andere Abstimmungskriterien anlegen. Im Vergleich zu Bevölkerungen sind Parlamentarier wohl eher in der Lage, eine relative Verbesserung gegenüber dem Status quo zu akzeptieren. Bei komplexen Ereignissen wie der Verfassungsgebung dürften Referenden deshalb eine größere Ablehnungsgefahr als Parlamentsentscheide bergen. Dieses Resultat könnte womöglich zu einer besseren Vermittlungsstrategie der Verhandlungsergebnisse führen, denn Information und Aufklärung sollten weniger an allgemeinen Bewertungen der Integration und Europas, als vielmehr am spezifischen Vergleich der wichtigsten Änderungen gegenüber dem Status quo ansetzen.

Schon heute und vor Abschluss des Verfassungsgebungsprozesses können wir anhand der DOSEI-Ergebnisse sagen, dass der Konvent eine sehr effektive Methode war, einem großen Kreis an Mitgliedsstaaten mit sehr unterschiedlichen Interessen ein Reformkonzept vorzulegen, das sich als Paket auf der folgenden Regierungskonferenz weitgehend durchsetzen konnte. Allerdings riskiert diese Methode, die sehr hohen Restriktionen der abschließenden Ratifikation zu vernachlässigen (König 2002). Was bei vorherigen Regierungskonferenzen »schwachen« Staaten zu Verhandlungsstärke verhalf und »unvorhersehbare « Ratifikationsrisiken ausschloss (Hug/König 2002), war bei den Laekener Verhandlungen nicht mehr zu beobachten. So dürfte ausgerechnet die auch von Deutschland geäußerte Forderung, das Paket nicht mehr aufschnüren zu wollen, die republikanische Idee zu Grabe getragen haben. Andererseits war diese normative Ausrichtung der Verfassungsgebung weder 
durch die vorherigen Parlamentswahlen noch den Konvent noch während der Ratifikationsdebatten thematisiert worden. Jedoch können wir festhalten, dass die Gipfelteilnehmer einem Entwurf zustimmten, der ihre Interessen besser als der Vertrag von Nizza repräsentiert. Interessanterweise vertraten Gipfelteilnehmer am effektivsten ihre nationalen Interessen, wenn Volksabstimmungen angekündigt waren (König/Finke 2005). Womöglich helfen Volksentscheide ein Delegationsproblem zu lösen, denn in einigen Fällen wichen die Gipfelteilnehmer ohne »Verhandlungsnotwendigkeit« von ihrem Auftrag zum Nachteil ihrer Prinzipale ab.

In Bezug auf die Integrationstheorien machen diese ersten DOSEI-Ergebnisse $\mathrm{m}$. E. deutlich, dass die Integrationsforschung einen Beitrag zur Erklärung historischer Ereignisse wie der Verfassungsgebung liefern kann. Es finden sich deutliche Effekte zwischen der nationalen und supranationalen Ebene, die teilweise von der Prozessdynamik überlagert oder sogar umgekehrt werden. Reformoptionen sind selbst unter 25 Mitgliedsstaaten aushandelbar, auch wenn die Lösung am Ende zu scheitern droht. Mit DOSEI liegt nun ein Archiv vor, das über mehrere Ereignisse hinweg nicht nur die beteiligten Akteure, sondern auch ihre Positionen zu den wichtigen Reformthemen erfasst. Schon heute können auf dieser Grundlage einige Kernaussagen der Integrationstheorien hinterfragt werden: erstens, ob sich die drei großen Staaten im intergouvernementalistischen Sinn durchsetzten konnten oder in eine rhetorische Falle verwickeln ließen. Zweitens, welche Rolle die supranationalen Akteure spielten und inwiefern sich Meinungen und Interessen anglichen. Drittens, welche Beziehung zwischen den Ebenen und welche Beziehung zwischen den Delegierten und ihren Prinzipalen bestand - unabhängig davon, ob es sich um kollektive Akteure wie Staaten oder Bevölkerungen, korporative Akteure wie Parteien oder Interessengruppen oder um individuelle Akteure wie die Delegierten des Konvents oder des Regierungsgipfels handelt.

Die in DOSEI erfassten Positionen reflektieren die Äußerungen und Einstellungen zu den inhaltlichen Fragen der Verfassungsgebung, die eine Vielzahl an Themen ins Gespräch gebracht haben. Im Gegensatz zum gemeinsamen Akteurskonzept dürfte m. E. die Interpretation dieser Positionen unter Integrationsforschern umstritten sein. Rationale Ansätze unterscheiden zwischen ehrlichen und strategischen Positionen, wohingegen andere Ansätze entweder die Stabilität der Positionen diskutieren oder die normative von der interessengeleiteten Unterfütterung unterscheiden. Vielleicht könnte der Vergleich der Prognosekraft der unterschiedlichen Ansätze ein erster Schritt auf dem Weg sein, mit ähnlichen Grundlagen die unterschiedlichen Annahmen dieser Ansätze zu überprüfen, die für die Erklärung der Ereignisse der Integration im Allgemeinen und der Verfassungsgebung bzw. der Republikgründung im Besonderen getroffen werden. 


\section{Literatur}

Gwiazda, Anna 2006: Poland: A Story of Success or Failure?, in: König/Hug 2006, i. E.

Hug, Simon/König, Thomas 2002: In View of Ratification: Governmental Preferences and Domestic Constraints at the Amsterdam Intergovernmental Conference, in: International Organization 56: 2, 447-476.

Hug, Simon/König, Thomas 2006: Conclusion, in: König/Hug 2006, i. E.

Hug, Simon/Schulz, Tobias 2005: Using Mass Survey Data to Infer Political Positions, in: European Union Politics 6: 3, 339-352.

König, Thomas 2002: Verfassungspolitik und Verfassungsdynamik (Vortrag auf der Tagung der Heidelberger Akademie der Wissenschaften und der Landesrektorenkonferenz zur Arbeit des Konvents zur Zukunft der Europäischen Union, Badisches Landesmuseum Karlsruhe, 21.10.2002), unv. Ms.

König, Thomas 2005: Methods and Analysing Positions on European Constitution-building, in: European Union Politics 6: 3, 259-267.

König, Thomas/Hug, Simon (Hrsg.) 2006: Policy-making Processes and the European Constitution: A Comparative Study of Member States and Accession Countries, London, i. E.

König, Thomas/Finke, Daniel/Daimer, Stephanie 2005: Ignoring the Non-Ignorables? Missingness and Missing Positions, in: European Union Politics 6: 3, 269-290.

König, Thomas/Finke, Daniel 2005: The (Il)loyal Agent: A Theoretical and Empirical Investigation into Delegates' Discretion in the Negotiations over a Constitution for Europe (Vortragspapier präsentiert auf dem ECPR Annual Meeting, Budapest, 7. - 10.9.2005), unv. Ms.

König, Thomas/Slapin, Jonathan 2005: Winning and Losing in European Constitution Building: Member States, Delegates and their Impact on the Constitutional Draft Treaty (Vortragspapier präsentiert auf dem ECPR Annual Meeting, Budapest, 7. - 10.9.2005), unv. Ms.

König, Thomas/Warntjen, Andreas/Burkhart, Simone 2006: The Laeken Convention: Consensus without Unity, in: König/Hug 2006, i. E.

Tsebelis, George 2006: The European Convention and the Rome and Brussels IGCs: A Veto Players Analysis, in: König/Hug 2006, i. E.

Tsebelis, George/Garrett, Geoffrey 2001: The Institutional Foundations of Intergovernmentalism and Supranationalism in the European Union, in: International Organization 55: 2, 357-390.

von Bogdandy, Armin 2005: Die europäische Republik, in: Aus Politik und Zeitgeschichte 36, 21-27. 